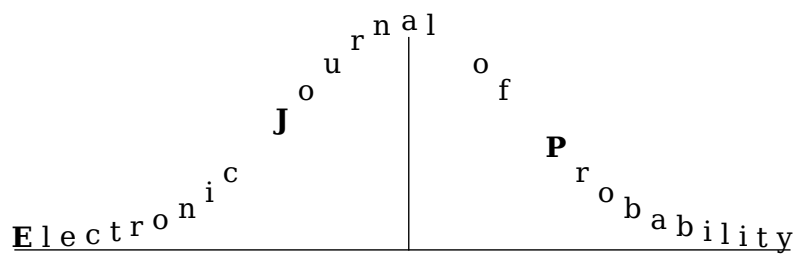

Electron. J. Probab. 26 (2021), article no. 101, 1-15.

ISSN: 1083-6489 https://doi.org/10.1214/21-EJP660

\title{
Gradient formulas for jump processes on manifolds*
}

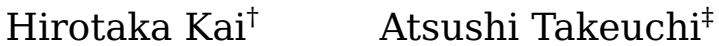 \\ Dedicated to Professor Takashi Komatsu on the occasion of his 76th birthday.
}

\begin{abstract}
Consider jump processes on a connected compact smooth Riemannian manifold, which are constructed by the canonical projection of the processes on the bundle of orthonormal frames. The condition under which the $M$-valued process is Markovian will be revisited as seen in Applebaum-Estrade [1]. Moreover, the gradient formula, which will be also called the integration by parts formula, can be also studied. The obtained formula can be regarded as the extended version of the celebrated Bismut formula on the case of diffusion processes.
\end{abstract}

Keywords: jump processes on manifolds; stochastic differential equations with jumps; integration by parts formulas.

MSC2020 subject classifications: $60 \mathrm{~J} 76 ; 58 \mathrm{~J} 65 ; 60 \mathrm{H} 07 ; 60 \mathrm{H} 10$.

Submitted to EJP on July 10, 2020, final version accepted on June 7, 2021.

\section{Introduction}

Stochastic differential equations on the Euclidean space have been quite well studied for a long time, and they have given us a lot of fruitful issues in wide areas. Moreover, as the natural interest on the study, it has been also done to extend our field of the equations to the ones on manifolds. Since the geometrical structure in each local coordinate of the manifold is the same as the Euclidean space, it seems us very natural to construct the process given by the equation by connecting the local solution to the equation in each local coordinate. We can also construct the process by rolling the manifold on the flat space along the inked trajectory of the process on the plane without slipping. This idea can be done by projecting the process valued in the bundle of the orthonormal frames over the manifold, which is determined by the stochastic differential equation on the bundle. In fact, the Brownian motion on the Riemannian manifold, whose infinitesimal generator is the Laplace-Beltrami operator, can be obtained by the projection of the horizontal Brownian motion on the bundle of the orthonormal frames over the manifold.

\footnotetext{
*This work was supported by JSPS KAKENHI Grant Number JP20K03641.

${ }^{\dagger}$ Osaka City University, Japan. E-mail: d20sa006@vt. osaka-cu.ac.jp

${ }^{\ddagger}$ Tokyo Woman’s Christian University, Japan. E-mail: a- takeuchi@lab. twcu.ac.jp
} 
The horizontal Brownian motion is the solution to the stochastic differential equation, and its infinitesimal generator is the horizontal Laplacian of Bochner. See Hsu [6] and Ikeda-Watanabe [8] on the detailed explanations.

It is very natural to consider whether the Lévy process on the manifold can be constructed or not, similarly to the case of the Brownian motion on the manifold. There are some studies: Hunt [7] studied the Lévy processes on Lie groups from the viewpoint of functional analysis, that is, the semigroup approach. Applebaum-Kunita [2] constructed the Lévy processes on Lie groups as the solutions to the jump-type stochastic differential equations, and studied the Lévy flows on manifolds. See also Fujiwara [5] and Kunita [11]. And Applebaum-Estrade [1] studied the isotropic Lévy processes on Riemannian manifolds. They proposed as one of sufficient conditions that the isotropic property on the Lévy measure of the driving process of the stochastic differential equation is required to the projecting procedure to define the manifold-valued process from the process valued in the bundle of the orthonormal frames.

In the present paper, we shall discuss the gradient formulas, which is often called the integration by parts formulas, for jump processes on Riemannian manifolds. In the case of the diffusion process without any jumps on the manifold, the formula is called the celebrated Bismut formula. The formula plays a crucial role in the study of the detailed property of the density function, and the study of the computations of the Greeks in mathematical finance. The Bismut formula can be obtained by some approaches: the Malliavin calculus on the Wiener space (cf. Nualart [12]) and the martingale method based upon the Kolmogorov backward equation of the corresponding infinitesimal generator of the diffusion process (cf. Elworthy-Li [4]). The martingale method is interpreted as the martingale representation theorem, or the Clark-Ocone formula which is famous in the study of mathematical finance. See also Bismut [3], and Hsu [6] and the references therein. Now, our strategy to attack the gradient formulas for jump processes on the manifold in the present paper is via the martingale approach. And our jump processes is defined by the projection of the jump-diffusion processes determined by the jump-type stochastic differential equation on the bundle of the orthonormal frames of the manifold, whose equation is often called the Marcus-type canonical stochastic differential equation of jump type (cf. Kunita [11] and Ishikawa [9]). In Takeuchi [15], the integration by parts formula for jump processes on the Euclidean space has been discussed, and our approach in the paper is almost parallel. Before doing it, we shall revisit the work on the Lévy process on the manifold by Applebaum-Estrade [1], in order to compare the obtained formula with the celebrated Bismut one for the diffusion process.

The organization of the paper is as follows: In Section 2, we shall prepare some notations and introduce the framework from the viewpoint of the differential geometry and the probability. The detailed description can be found in Kobayashi-Nomizu [10] and Sakai [13] on the differential geometry, and in Hsu [6] and Ikeda-Watanabe [8] on the probability. Moreover, the result by Applebaum-Estrade [1] on the sufficient condition, under which the $M$-valued process projected by the $O(M)$-valued process is Markovian, is revisited in Proposition 2.2. Section 3 is the main part of the present paper. The main results on the gradient formulas about the $M$-valued process are mentioned, according to the situations focused on the effects by the diffusion term, the jump one and the hybrid one. Especially, the first result (Theorem 3.3) can be also interpreted as the extension of the celebrated Bismut formula on the $M$-valued diffusion process without any jumps, as introduced in Bimut [3] and Hsu [6]. Another two results (Theorems 3.10 and 3.12) are very interesting in the sense that those formulas are focused by the effects of the jump term in the process. Those results will be proved in Section 4 . The strategy to attack the proofs are almost parallel to the paper by Takeuchi [15] on the Euclidean space. 


\section{Preliminaries}

First of all, we shall prepare some notations from differential geometry. Details can be seen in Kobayashi-Nomizu [10] and Sakai [13]. Let $(M, g)$ be a connected, compact and smooth Riemannian manifold of dimension $m$ with the Levi-Civita connection $\nabla=$ $\left\{\Gamma_{j k}^{i} ; 1 \leq i, j, k \leq m\right\}$. And let $\mathfrak{X}(M)$ or $\mathfrak{X}(O(M))$ the family of $C^{\infty}$-vector fields over $M$, respectively $O(M)$. Denote the bundle of linear frames on $M$ by

$$
G L(M)=\left\{r=\left(x, e_{x}\right) ; x \in M, e_{x}=\left(\left(e_{1}\right)_{x}, \ldots,\left(e_{m}\right)_{x}\right) \text { is a basis in } T_{x} M\right\} .
$$

Remark that in a local coordinate $\left(x_{i}, e_{i}^{j} ; 1 \leq i, j \leq m\right)$, each $\left(e_{i}\right)_{x} \in T_{x} M$ can be expressed as

$$
\left(e_{i}\right)_{x}=\sum_{j=1}^{m} e_{i}^{j}\left(\frac{\partial}{\partial x_{j}}\right)_{x} .
$$

Define the submanifold of $G L(M)$ by

$$
\begin{array}{r}
O(M)=\left\{r=\left(x, e_{x}\right) \in G L(M) ; e_{x}=\left(\left(e_{1}\right)_{x}, \ldots,\left(e_{m}\right)_{x}\right)\right. \\
\text { is an orthonormal basis in } \left.T_{x} M\right\},
\end{array}
$$

which is called the bundle of orthonormal frames on $M$. Remark that $O(M)$ is compact, because so is $M$. The canonical projection $\pi: O(M) \rightarrow M$ is defined by $\pi(r)=x$ for $r=\left(x, e_{x}\right) \in O(M)$. Let $H_{1}, \ldots, H_{m}$ be in $\mathfrak{X}(G L(M))$ such that $H_{i}(r)$ is the horizontal lift of $\left(e_{i}\right)_{x} \in T_{x} M$ for each $r=\left(x, e_{x}\right) \in O(M)$ and $1 \leq i \leq m$, which are often called the canonical horizontal vector fields in $O(M)$. In a local coordinate $\left(x_{i}, e_{i}^{j} ; 1 \leq i, j \leq m\right)$, those vector fields are written as follows:

$$
H_{i}=\sum_{j=1}^{m} e_{i}^{j} \frac{\partial}{\partial x_{j}}-\sum_{k, l, p, q=1}^{m} \Gamma_{k l}^{q}(x) e_{i}^{k} e_{p}^{l} \frac{\partial}{\partial e_{p}^{q}}, \quad 1 \leq i \leq m .
$$

Write $H=\left(H_{1}, \ldots, H_{m}\right)$ for the sake of simplicity. Finally, we shall give a small remark that

Remark 2.1. For $f \in C^{\infty}(M ; \mathbb{R})$ and $r=\left(x, e_{x}\right) \in O(M)$, it holds that

$$
(H(f \circ \pi))(r)=(e f)(x) .
$$

Write $\mathbb{R}_{0}^{m}=\mathbb{R}^{m} \backslash\{\mathbf{0}\}$. For given $z \in \mathbb{R}_{0}^{m}$, let $\Xi^{z}=\left\{\Xi^{z, \sigma}(r) ; \sigma \in[0,1], r \in O(M)\right\}$ be the one parameter group of diffeomorphisms over $O(M)$, which is often denoted by $\Xi^{z, \sigma}(r)=\operatorname{Exp}[\sigma H z](r)$, that is, the unique solution to the ordinary differential equation of the form:

$$
\frac{d}{d \sigma} \Xi^{z, \sigma}(\cdot)=(H z)\left(\Xi^{z, \sigma}(\cdot)\right), \quad \Xi^{z, 0}(\cdot)=i d_{O(M)}(\cdot),
$$

where $i d_{O(M)}: O(M) \rightarrow O(M)$ is the identity. More precisely, the equation (2.3) means that for any $F \in C^{\infty}(O(M) ; \mathbb{R})$,

$$
F\left(\Xi^{z, \sigma}(r)\right)=F(r)+\int_{0}^{\sigma}((H z) F)\left(\Xi^{z, u}(r)\right) d u .
$$

Denote by $\Xi_{*}^{z}=\left\{\Xi_{*}^{z, \sigma}(r) ; \sigma \in[0,1], r \in O(M)\right\}$ the family of the derivatives of the diffeomorphism $\Xi^{z, \sigma}(\cdot): O(M) \rightarrow O(M)$ for each $z \in \mathbb{R}_{0}^{m}$ and $\sigma \in[0,1]$. Then, the family $\Xi_{*}^{z}(r)$ satisfies the equation

$$
\frac{d}{d \sigma} \Xi_{*}^{z, \sigma}(\cdot)=\left((H z)_{*}\left(\Xi^{z, \sigma}(\cdot)\right)\right) \circ\left(\Xi_{*}^{z, \sigma}(\cdot)\right), \quad \Xi_{*}^{z, 0}(\cdot)=i d_{O(M)}(\cdot) .
$$


And, the diffeomorphic property of the mapping $\Xi^{z}$ tells us to see that $\left(\Xi_{*}^{z, \sigma}(\cdot)\right)^{-1}=$ $\Xi_{*}^{-z, \sigma}(\cdot)$, and that the inverse satisfies the equation

$$
\frac{d}{d \sigma}\left(\Xi_{*}^{z, \sigma}(\cdot)\right)^{-1}=-\left(\Xi_{*}^{z, \sigma}(\cdot)\right)^{-1} \circ\left((H z)_{*}\left(\Xi_{\sigma}^{z}(\cdot)\right)\right), \quad\left(\Xi_{*}^{z, 0}(\cdot)\right)^{-1}=i d_{O(M)}(\cdot) .
$$

Moreover, the $z$-derivative $\partial_{z} \Xi^{z}=\left\{\partial_{z} \Xi^{z, \sigma}(r) ; \sigma \in[0,1], r \in O(M)\right\}$ satisfies the equation

$$
\frac{d}{d \sigma} \partial_{z} \Xi^{z, \sigma}(\cdot)=(H z)_{*}\left(\Xi^{z, \sigma}(\cdot)\right) \circ \partial_{z} \Xi^{z, \sigma}(\cdot)+H\left(\Xi^{z, \sigma}(\cdot)\right), \quad \partial_{z} \Xi^{z, 0}(\cdot)=\mathbf{0} .
$$

Then, it can be solved as

$$
\partial_{z} \Xi^{z, \sigma}(\cdot)=\int_{0}^{\sigma}\left(\Xi_{*}^{z, \sigma}(\cdot)\right) \circ\left(\Xi_{*}^{z, u}(\cdot)\right)^{-1} \circ H\left(\Xi^{z, u}(\cdot)\right) d u .
$$

Moreover, we see from Proposition 6.3 of [10] that, for each $z \in \mathbb{R}_{0}^{m}$, the curve $\xi^{z}=$ $\left\{\xi^{z, \sigma}(x) ; \sigma \in[0,1], x \in M\right\}$ defined by

$$
\xi^{z, \sigma}(x):=\pi\left(\Xi^{z, \sigma}(r)\right), \quad r=(x, e) \in O(M)
$$

is the geodesic on $M$.

Now, we shall proceed our position to introduce the probabilistic setting in our framework. Let $T>0$ be fixed throughout the paper. Denote by $\nu(d z)$ a Lévy measure over $\mathbb{R}_{0}^{m}$ such that

$$
\int_{\mathbb{R}_{0}^{m}}\left(|z|^{2} \wedge 1\right) \nu(d z)<+\infty .
$$

On a probability space $(\Omega, \mathcal{F}, \mathbb{P})$, let $B=\left\{B_{t}=\left(B_{t}^{1}, \ldots, B_{t}^{m}\right) ; t \in[0, T]\right\}$ be an $m$ dimensional Brownian motion with $B_{0}=0 \in \mathbb{R}^{m}$, and $J=\left\{J_{t} ; t \in[0, T]\right\}$ the $\mathbb{R}^{m}$-valued pure-jump Lévy process defined by

$$
J_{t}=\int_{0}^{t} \int_{\mathbb{R}_{0}^{m}} z \bar{N}(d s, d z),
$$

where $N(d t, d z)$ is a Poisson random measure on $[0, T] \times \mathbb{R}_{0}^{m}$ with the intensity $\hat{N}(d t, d z)=$ $d t \nu(d z), \tilde{N}(d t, d z)=N(d t, d z)-\hat{N}(d t, d z)$ is the compensated Poisson random measure. For the sake of simplicity of notations, write

$$
\bar{N}(d t, d z)=\mathbb{I}_{(|z| \leq 1)} \tilde{N}(d t, d z)+\mathbb{I}_{(|z|>1)} N(d t, d z) .
$$

For $r=\left(x, e_{x}\right) \in O(M)$, let $R=\left\{R_{t} ; t \in[0, T]\right\}$ be the $O(M)$-valued process determined by the jump-type stochastic differential equation on $O(M)$ of the form:

$$
d R_{t}=H\left(R_{t}\right) \circ d B_{t}+\int_{\mathbb{R}_{0}^{m}}\left\{\Xi^{z, 1}\left(R_{t-}\right)-R_{t-}\right\} \bar{N}(d t, d z), \quad R_{0}=r,
$$

where $\circ d B_{t}$ is the Stratonovich stochastic integral with respect to the $m$-dimensional Brownian motion $B$. More precisely, the equation (2.10) should be written as

$$
\begin{aligned}
F\left(R_{t}\right)= & F(r)+\int_{0}^{t}(H F)\left(R_{s}\right) \circ d B_{s} \\
& +\int_{0}^{t} \int_{\mathbb{R}_{0}^{m}}\left\{F\left(\Xi^{z, 1}\left(R_{s-}\right)\right)-F\left(R_{s-}\right)\right\} \bar{N}(d s, d z) \\
& +\int_{0}^{t} \int_{\mathbb{R}_{0}^{m}}\left\{F\left(\Xi^{z, 1}\left(R_{s}\right)\right)-F\left(R_{s}\right)-((H z) F)\left(R_{s}\right) \mathbb{I}_{(|z| \leq 1)}\right\} \hat{N}(d s, d z)
\end{aligned}
$$


for all $F \in C^{\infty}(O(M) ; \mathbb{R})$ with a compact support. In our situation, there exists the pathwise unique solution to the equation (2.10) such that the process lies in $O(M)$, and that the explosion time of the process is infinity a.s. (cf. Kunita [11]-Proposition 7.1.1). In order to emphasize the dependence on the initial point $r \in O(M)$ of the equation (2.10), we shall also write $R(r)=\left\{R_{t}(r) ; t \in[0, T]\right\}$. Then, we can easily check that the $O(M)$-valued process $R$ is Markovian, and its infinitesimal generator $\mathcal{A}$ is

$$
\begin{aligned}
(\mathcal{A} F)(r)= & \frac{1}{2} \sum_{i=1}^{m}\left(H_{i}\left(H_{i} F\right)\right)(r) \\
& +\int_{\mathbb{R}_{0}^{m}}\left\{F\left(\Xi^{z, 1}(r)\right)-F(r)-((H z) F)(r) \mathbb{I}_{(|z| \leq 1)}\right\} \nu(d z)
\end{aligned}
$$

for $F \in C^{\infty}(O(M) ; \mathbb{R})$ with a compact support. See Applebaum-Kunita [2], Fujiwara [5] and Kunita [11] on the detailed studies.

Recall that $x=\pi(r) \in M$ for $r=\left(x, e_{x}\right) \in O(M)$, and define the $M$-valued process $X=\left\{X_{t} ; t \in[0, T]\right\}$ by $X_{t}=\pi\left(R_{t}\right)$. Then, for $f \in C^{\infty}(M ; \mathbb{R})$, we see from (2.11) that

$$
\begin{aligned}
f\left(X_{t}\right)= & (f \circ \pi)\left(R_{t}\right) \\
= & (f \circ \pi)(r)+\int_{0}^{t}(H(f \circ \pi))\left(R_{s}\right) \circ d B_{s} \\
& +\int_{0}^{t} \int_{\mathbb{R}_{0}^{m}}\left\{(f \circ \pi)\left(\Xi^{z, 1}\left(R_{s-}\right)\right)-(f \circ \pi)\left(R_{s-}\right)\right\} \bar{N}(d s, d z) \\
& +\int_{0}^{t} \int_{\mathbb{R}_{0}^{m}}\left\{(f \circ \pi)\left(\Xi^{z, 1}\left(R_{s}\right)\right)-(f \circ \pi)\left(R_{s}\right)-((H z)(f \circ \pi))\left(R_{s}\right) \mathbb{I}_{(|z| \leq 1)}\right\} \hat{N}(d s, d z) \\
= & f(x)+\int_{0}^{t}\left(\left(\pi_{*} H\right) f\right)\left(X_{s}\right) \circ d B_{s}+\int_{0}^{t} \int_{\mathbb{R}_{0}^{m}}\left\{f\left(\xi^{z, 1}\left(X_{s-}\right)\right)-f\left(X_{s-}\right)\right\} \bar{N}(d s, d z) \\
& +\int_{0}^{t} \int_{\mathbb{R}_{0}^{m}}\left\{f\left(\xi^{z, 1}\left(X_{s}\right)\right)-f\left(X_{s}\right)-\left(\left(\left(\pi_{*} H\right) z\right) f\right)\left(X_{s}\right) \mathbb{I}_{(|z| \leq 1)}\right\} \hat{N}(d s, d z),
\end{aligned}
$$

that is, the $M$-valued process $X=\left\{X_{t} ; t \in[0, T]\right\}$ satisfies the following equation:

$$
d X_{t}=\left(\pi_{*} H\right)\left(X_{t}\right) \circ d B_{t}+\int_{\mathbb{R}_{0}^{m}}\left\{\xi^{z, 1}\left(X_{t-}\right)-X_{t-}\right\} \bar{N}(d t, d z), \quad X_{0}=x .
$$

Remark that the $M$-valued process $X$ depend on the initial point $r=\left(x, e_{x}\right) \in O(M)$ of the equation (2.10), in general, because so do the push-forward vector fields $\pi_{*} H_{i}(1 \leq i \leq$ $m)$. We shall also denote the solution to the equation (2.13) by $\left\{X_{t}\left(\left(x, e_{x}\right),(B, J)\right) ; t \in\right.$ $[0, T]\}$, in order to emphasize the dependence of the initial point $\left(x, e_{x}\right)$ and the driving processes $(B, J)$. Define the linear operator $\mathcal{L}$ by

$$
\begin{aligned}
(\mathcal{L} f)(x)= & \frac{1}{2} \sum_{i=1}^{m}\left(\left(\pi_{*} H_{i}\right)\left(\left(\pi_{*} H_{i}\right) f\right)\right)(x) \\
& +\int_{\mathbb{R}_{0}^{m}}\left\{f\left(\xi^{z, 1}(x)\right)-f(x)-\left(\left(\left(\pi_{*} H\right) z\right) f\right)(x) \mathbb{I}_{(|z| \leq 1)}\right\} \nu(d z) .
\end{aligned}
$$

Since the solution process $X$ and the operator $\mathcal{L}$ depend on the choice of the initial point $r=\left(x, e_{x}\right) \in O(M)$ of the equation (2.10), the process $X$ is not always Markovian.

Proposition 2.2 (cf. [1]-Theorem 3.1). Suppose that the measure $\nu(d z)$ is rotationally invariant, that is, $\nu(K)=\nu(A K)$ for $K \in \mathcal{B}\left(\mathbb{R}_{0}^{m}\right)$ and $A \in O(m, \mathbb{R})$, where $O(m, \mathbb{R}) \subset$ 
$\mathbb{R}^{m} \otimes \mathbb{R}^{m}$ is the family of orthogonal matrices, and $A K=\{A x ; x \in K\}$ is the image of the set $K \subset \mathbb{R}_{0}^{m}$ by $A \in \mathbb{R}^{m} \otimes \mathbb{R}^{m}$. Then, for each $t \in[0, T]$,

$$
X_{t}\left(\left(x, e_{x} A\right),(B, J)\right) \stackrel{\mathcal{L}}{=} X_{t}\left(\left(x, e_{x}\right),(A B, A J)\right) .
$$

Proof. For an orthogonal matrix $A \in O(m, \mathbb{R})$, we shall write

$$
e_{x} A=\left(\left(e_{x} A\right)_{1}, \ldots,\left(e_{x} A\right)_{m}\right), \quad\left(e_{x} A\right)_{i}=\sum_{j=1}^{m}\left(e_{j}\right)_{x} A_{j i} .
$$

Then, since the $m$-dimensional Brownian motion $B$ and the Lévy measure $\nu(d z)$ over $\mathbb{R}_{0}^{m}$ are rotationally invariant, the process $(A B, A J)=\left\{\left(A B_{t}, A J_{t}\right) ; t \in[0, T]\right\}$ has the same law as $(B, J)$, which implies that

$$
X_{t}\left(\left(x, e_{x}\right),(A B, A J)\right) \stackrel{\mathcal{L}}{=} X_{t}\left(\left(x, e_{x}\right),(B, J)\right) .
$$

On the other hand, the uniqueness of the solutions to the equation (2.13) tells us to see that

$$
X_{t}\left(\left(x, e_{x}\right),(A B, A J)\right) \stackrel{\mathcal{L}}{=} X_{t}\left(\left(x, e_{x} A\right),(B, J)\right) .
$$

Hence, combining these two results leads us to get the conclusion.

Example 2.3. The Lévy measures of the stable process and the truncated stable process are typical examples with rotationally invariance property.

\section{Results}

In this section, let us introduce our results of the paper, which will be proved in the next section. Write

$$
\left(T_{t} f\right)(x):=\mathbb{E}\left[f\left(X_{t}(x)\right)\right], \quad\left(S_{t} F\right)(r):=\mathbb{E}\left[F\left(R_{t}(r)\right)\right]
$$

for $f: M \rightarrow \mathbb{R}$ and $F: O(M) \rightarrow \mathbb{R}$ with nice properties on the boundedness and the regularity. Here, we have used the expressions $X_{t}(x)$ and $R_{t}(r)$, in order to emphasize the dependence on the initial points $x$ and $r$ of the processes $X$ and $R$, respectively. It can be easily checked that the family $\left\{S_{t} ; t \in[0, T]\right\}$ is the Feller semigroup with the infinitesimal generator $\mathcal{A}$. Furthermore, under the rotationally invariant setting on the measure $\nu(d z)$, the family $\left\{T_{t} ; t \in[0, T]\right\}$ is also the Feller semigroup with the infinitesimal generator $\mathcal{L}$ (cf. Applebaum-Estrade [1]). Our main interest here is to construct the gradient formula

$$
\left(e\left(T_{t} f\right)\right)(x)=\mathbb{E}\left[f\left(X_{t}(x)\right) \Gamma_{t}\right]
$$

in the situation where the measure $\nu(d z)$ is not always rotationally invariant, where $e_{x}=\left(\left(e_{1}\right)_{x}, \ldots,\left(e_{m}\right)_{x}\right)$ is the orthonormal basis in $T_{x} M$. So, we don't know whether the $M$-valued process $X$ is Markovian or not. The formula raised above is also called the celebrated Bismut formula for the diffusion process on $M$ without any jumps, that is, the $M$-valued Brownian motion with the infinitesimal generator $\triangle_{M} / 2$, where $\triangle_{M}$ is the Laplace-Beltrami operator on $M$. See Hsu [6]-Theorems 8.3.3 and 8.3.4 on the details.

At the beginning, we shall add the additional condition on the Lévy measure $\nu(d z)$, in order to guarantee the higher-order moments of the processes in our framework.

Assumption 3.1. Suppose that $\int_{|z|>1}|z|^{p} \nu(d z)<+\infty$ for all $p \geq 1$. 
Example 3.2. The Lévy measure of the truncated stable process, and the product one of the 1-dimensional gamma processes or the 1-dimensional tempered stable processes, satisfy Assumption 3.1.

Recall that the operator $\mathcal{A}$ given by (2.12) is the infinitesimal generator of the process $R$, and write $[\mathcal{A}, V]=\mathcal{A} V-V \mathcal{A}$ for $V \in \mathfrak{X}(O(M))$. Let $\left\{L_{t} ; t \in[0, T]\right\}$ be the $\mathbb{R}^{m} \otimes \mathbb{R}^{m}$ valued function determined by the ordinary differential equation of the form:

$$
\frac{d L_{t}}{d t}=-L_{t} C\left(R_{t}\right), \quad L_{0}=I_{m},
$$

where $I_{m}$ is the identity, $H_{k}^{*}(r) \in T_{r}^{*} O(M)$ is the dual of $H_{k}(r) \in T_{r} O(M)$, and

$$
C(r)=\left(\begin{array}{ccc}
\left(H_{1}^{*}\left(\left[\mathcal{A}, H_{1}\right]\right)\right)(r) & \cdots & \left(H_{m}^{*}\left(\left[\mathcal{A}, H_{1}\right]\right)\right)(r) \\
\vdots & & \vdots \\
\left(H_{1}^{*}\left(\left[\mathcal{A}, H_{m}\right]\right)\right)(r) & \cdots & \left(H_{m}^{*}\left(\left[\mathcal{A}, H_{m}\right]\right)\right)(r)
\end{array}\right)
$$

for $r \in O(M)$.

Theorem 3.3. Let $0<t \leq T$. Then, under Assumption 3.1, it holds that

$$
\left(e\left(T_{t} f\right)\right)(x)=\mathbb{E}\left[f\left(X_{t}(x)\right) \frac{I_{t}}{t}\right]
$$

for $f \in C^{\infty}(M ; \mathbb{R})$, where $I_{t}:=\int_{0}^{t}\left(L_{s} d B_{s}\right)^{*}$.

Remark 3.4. The equation (3.2) in Theorem 3.3 is exactly the same representation as the celebrated Bismut formula for the Brownian motion on $M$ (cf. Hsu [6]-Theorems 8.3.3 and 8.3.4).

Theorem 3.3 is the result on the gradient formula focused on the diffusion term in the equation (2.13) only. Since (2.13) also has the jump term, our next interest is to discuss the problem whether the effect of the jump term can be included in the gradient formula or not. Now, we shall proceed our position to discuss such a problem. Before doing it, we shall add the following conditions on the measure $\nu(d z)$ :

Assumption 3.5. Suppose that

(i) there exists $\alpha>0$ such that $\liminf _{\rho \searrow 0} \rho^{\alpha} \int_{\mathbb{R}_{0}^{m}}\left(\left|\frac{z \cdot \theta}{\rho}\right|^{2} \wedge 1\right) \nu(d z)>0$,

(ii) the measure $\nu(d z)$ has the smooth density $g(z)$ with respect to the Lebesgue measure on $\mathbb{R}_{0}^{m}$ such that $\lim _{|z| \rightarrow+\infty}|z|^{2} g(z)=0$.

Remark 3.6. Whenever the regularity of the density for the solution to a stochastic differential equation of jump type in the Euclidean space is discussed, we often see the conditions on the measure $\nu(d z)$ :

(a) the order condition: there exists a constant $0<\beta<2$ such that

$$
\liminf _{\rho \searrow 0} \rho^{-\beta} \int_{|z| \leq \rho}|z|^{2} \nu(d z)>0,
$$

(b) the non-degenerate condition: there exists a positive definite matrix $\Theta \in \mathbb{R}^{m} \otimes \mathbb{R}^{m}$ such that

$$
\liminf _{\rho \searrow 0}\left(\int_{|z| \leq \rho}|z|^{2} \nu(d z)\right)^{-1} \int_{|z| \leq \rho}|z \cdot \theta|^{2} \nu(d z) \geq \theta \cdot \Theta \theta
$$

for any $\theta \in \mathbb{R}^{m}$ with $|\theta|=1$. 
See Ishikawa [9] on the detailed statements. The conditions (a) and (b) stated above implies the condition (i) in Assumption 3.5. In fact, it is easy to check that

$$
\int_{\mathbb{R}_{0}^{m}}\left(\left|\frac{z \cdot \theta}{\rho}\right|^{2} \wedge 1\right) \nu(d z) \geq \rho^{-2} \int_{|z| \leq \rho}|z \cdot \theta|^{2} \nu(d z) \geq c \rho^{-2+\beta} \theta \cdot \Theta \theta .
$$

Moreover, we shall suppose the geometric condition as follows:

Assumption 3.7. The manifold $M$ is simply connected and has the non-positive sectional curvature.

Remark 3.8. Since the manifold $M$ is compact, so is the orthonormal frame bundle $O(M)$. Hence, the vector fields $\left\{H_{i} ; 1 \leq i \leq m\right\}$ on $O(M)$ are complete. Then, the Cartan-Hadamard theorem yields that the mapping $\mathbb{R}_{0}^{m} \ni z \longmapsto \xi^{z, \sigma}(x)=\pi\left(\Xi^{z, \sigma}(r)\right) \in M$ is the diffeomorphism for each $r=(x, e) \in O(M)$ and $\sigma \in[0,1]$. See Sakai [13] on the detailed statement.

Then, taking the (partial) derivative of (2.9) in $z \in \mathbb{R}_{0}^{m}$ implies that

$$
\left(\pi_{*}\left(\Xi^{z, \sigma}(r)\right)\right) \circ\left(\partial_{z} \Xi^{z, \sigma}(r)\right)=\partial_{z} \xi^{z, \sigma}(\pi(r)) .
$$

Since the inverse of $\partial_{z} \xi^{z, \sigma}(r)$ is well-defined as stated above, we see that the inverse of $\partial_{z} \Xi^{z, \sigma}(r)$ is also well-defined, and that

$$
\left(\partial_{z} \Xi^{z, \sigma}(r)\right)^{-1}=\left\{\partial_{z} \xi^{z, \sigma}(r)\right\}^{-1} \circ\left(\pi_{*}\left(\Xi^{z, \sigma}(r)\right)\right) .
$$

Remark 3.9. We shall give a remark on the case where the manifold $M$ doesn't always satisfy Assumption 3.7. Then, we need another condition on the Lévy measure $\nu(d z)$ such that the support of the measure $\nu(d z)$ is included in the closed ball centered at the origin with the injectivity radius of the manifold $M$. Remark that the injectivity radius is strictly positive in our such situation. Hence, the inverse of $\partial_{z} \xi^{z, \sigma}(r)$ is well-defined on the support of the measure $\nu(d z)$, and so is the inverse of $\partial_{z} \Xi^{z, \sigma}(r)$.

Recall that $x \in M$ is the initial point of the process $X$. Let $h\left(=h^{x}\right):[0, T] \times \mathbb{R}_{0}^{m} \rightarrow$ $[0,+\infty)$ be deterministic such that $h(t, \cdot) \in C_{b}^{1}\left(\mathbb{R}_{0}^{m} ;[0,+\infty)\right)$ for each $t \in[0, T]$, and that $h(t, z) \leq C_{T}\left(|z|^{2} \wedge 1\right)$, where $C_{T}$ is the positive constant depending on $T$. Let us introduce the following notations:

$$
\begin{aligned}
& A_{t}=\int_{0}^{t} \int_{\mathbb{R}_{0}^{m}} h(s, z) N(d s, d z), \quad v(s, z)=\left(\partial_{z} \Xi^{z, 1}\left(R_{s}\right)\right)^{-1} \circ\left(H\left(\Xi^{z, 1} \circ R_{s}\right)\right)(r) h(s, z), \\
& J_{t}=\int_{0}^{t} \int_{\mathbb{R}_{0}^{m}} \frac{\operatorname{div}_{z}(g(z) v(s, z))}{g(z)} \tilde{N}(d s, d z), \quad K_{t}=\int_{0}^{t} \int_{\mathbb{R}_{0}^{m}} \partial_{z} h(s, z) v(s, z) N(d s, d z) .
\end{aligned}
$$

Theorem 3.10. Let $0<t \leq T$. Then, under Assumptions 3.1, 3.5 and 3.7, it holds that, for $f \in C^{\infty}(M ; \mathbb{R})$,

$$
\left(e\left(T_{t} f\right)\right)(x)=\mathbb{E}\left[f\left(X_{t}\right)\left(-\frac{J_{t}}{A_{t}}+\frac{K_{t}}{A_{t}^{2}}\right)\right] .
$$

Remark 3.11. From the property on the function $h$, we can check, similarly to Remark 3.2 in Takeuchi [15], that $1 / A_{t} \in \mathbb{L}^{p}(\Omega)$ for any $p>1$. In fact, define

$$
M_{t}^{\lambda}:=\int_{0}^{t} \int_{\mathbb{R}_{0}^{m}}\{\exp (-\lambda h(s, z))-1\} \hat{N}(d s, d z) .
$$

Then, we see that

$$
\mathbb{E}\left[A_{t}^{-p}\right]=\frac{1}{\Gamma(p)} \int_{0}^{+\infty} \lambda^{p-1} \mathbb{E}\left[\exp \left(-\lambda A_{t}\right)\right] d \lambda
$$




$$
\begin{aligned}
& =\frac{1}{\Gamma(p)} \int_{0}^{+\infty} \lambda^{p-1} \mathbb{E}\left[\exp \left(-\lambda A_{t}-M_{t}^{\lambda}\right)\right] \exp \left(M_{t}^{\lambda}\right) d \lambda \\
& \leq \frac{1}{\Gamma(p)} \int_{0}^{+\infty} \lambda^{p-1} \exp \left\{-C_{T} \lambda \int_{\mathbb{R}_{0}^{m}}\left(|z|^{2} \wedge 1\right) \nu(d z)\right\} d \lambda<+\infty,
\end{aligned}
$$

where $\Gamma(p)=\int_{0}^{+\infty} x^{p-1} e^{-x} d x$ is the usual gamma function.

Theorem 3.10 is only focused on the effect of the jump term in the equation (2.10) or (2.13). We can also derive the hybrid result on the diffusion and the jump terms as follows:

Theorem 3.12. Let $0<t \leq T$. Then, under Assumptions 3.1, 3.5 and 3.7, it holds that, for $f \in C^{\infty}(M ; \mathbb{R})$,

$$
\left(e\left(T_{t} f\right)\right)(x)=\mathbb{E}\left[f\left(X_{t}\right)\left(\frac{I_{t}-J_{t}}{t+A_{t}}+\frac{K_{t}}{\left(t+A_{t}\right)^{2}}\right)\right] .
$$

Remark 3.13. Since

$$
\operatorname{dim} \mathfrak{L}\left[\left(\pi_{*} H_{i}\right)(x) ; 1 \leq i \leq m\right]=\operatorname{dim} \mathfrak{L}\left[e_{i}(x) ; 1 \leq i \leq m\right]=m
$$

for any $x \in M$, where $\mathfrak{L}\left[a_{i} ; 1 \leq i \leq m\right]$ represents the linear space spanned by the vectors $a_{1}, \ldots, a_{m} \in T_{x} M$, we can justify from Theorems 3.3, 3.10 and 3.12 that, for each $0<t \leq T$, the probability law of the $M$-valued random variable $X_{t}$ is absolutely continuous with respect to the volume element on $M$, via the Sobolev-type inequality. See Takeuchi [14] and Taniguchi [16] on the detailed argument.

\section{Proofs}

In this section, we shall give the sketch of the proofs on the results raised in the previous section only. Our strategy is almost parallel to the method in Takeuchi [15] on the study of the integration by parts formula in the case of the Euclidean space.

For $f \in C^{2}(M ; \mathbb{R})$ with a compact support, define

$$
\Phi(s, r):=\mathbb{E}\left[(f \circ \pi)\left(R_{t-s}(r)\right)\right]
$$

for $s \in[0, t)$ and $r=(x, e) \in O(M)$. Then, we see that $\Phi \in C_{b}^{1,2}([0, t) \times O(M) ; \mathbb{R})$, and that the Kolmogorov backward equation

$$
\left(\frac{\partial \Phi}{\partial s}\right)(s, r)+(\mathcal{A} \Phi)(s, r)=0,(s \in[0, t)) \quad \lim _{s \nearrow t} \Phi(s, r)=f(x)
$$

is satisfied. Hence, the Itô formula leads us to obtain that, for $s \in[0, t)$,

$$
\begin{aligned}
\Phi\left(s, R_{s}\right)= & \Phi(0, r)+\int_{0}^{s}(H \Phi)\left(u, R_{u}\right) d B_{u} \\
& +\int_{0}^{s} \int_{\mathbb{R}_{0}^{m}}\left\{\Phi\left(u, \Xi^{z, 1}\left(R_{u-}\right)\right)-\Phi\left(u, R_{u-}\right)\right\} \tilde{N}(d u, d z) .
\end{aligned}
$$

Taking the limit as $s \nearrow t$ implies that

$$
\begin{aligned}
f\left(X_{t}\right)= & \mathbb{E}\left[f\left(X_{t}\right)\right]+\int_{0}^{t}(H \Phi)\left(u, R_{u}\right) d B_{u} \\
& +\int_{0}^{t} \int_{\mathbb{R}_{0}^{m}}\left\{\Phi\left(u, \Xi^{z, 1}\left(R_{u-}\right)\right)-\Phi\left(u, R_{u-}\right)\right\} \tilde{N}(d u, d z),
\end{aligned}
$$

because of $f \in C^{2}(M ; \mathbb{R})$ with a compact support. 


\subsection{Proof of Theorem 3.3}

In this subsection, let us give the proof of Theorem 3.3. Before doing it, we shall prepare the following lemma, which will play a key role in the argument.

Lemma 4.1. For $s \in[0, t]$ and $1 \leq i \leq m$, it holds that

$$
\begin{aligned}
\sum_{j=1}^{m} L_{s}^{i j}\left(H_{j} \Phi\right) & \left(s, R_{s}\right)=\left(H_{i} \Phi\right)(0, r)+\int_{0}^{s} \sum_{j, k=1}^{m} L_{u}^{i j}\left(H_{k} H_{j} \Phi\right)\left(u, R_{u}\right) d B_{u}^{k} \\
+ & \int_{0}^{s} \int_{\mathbb{R}_{0}^{m}} \sum_{j=1}^{m} L_{u-}^{i j}\left\{\left(H_{j} \Phi\right)\left(u, \Xi^{z, 1}\left(R_{u-}\right)\right)-\left(H_{j} \Phi\right)\left(u, R_{u-}\right)\right\} \tilde{N}(d u, d z) .
\end{aligned}
$$

Proof. Let $s \in[0, t), 1 \leq j \leq m$, and $\left[V_{1}, V_{2}\right]=V_{1} V_{2}-V_{2} V_{1}$ for $V_{1}, V_{2} \in \mathfrak{X}(O(M))$. Then, since $\left(\left[(z H), H_{i}\right] \Phi\right)(u, r)=0$ for $1 \leq i \leq m$, we have

$$
\begin{aligned}
\left(\left[\mathcal{A}, H_{j}\right] \Phi\right)\left(u, R_{u}\right)= & \frac{1}{2} \sum_{i=1}^{m}\left(\left[H_{i} H_{i}, H_{j}\right] \Phi\right)\left(u, R_{u}\right) \\
& +\int_{\mathbb{R}_{0}^{m}}\left\{\left(H_{j} \Phi\right)\left(u, \Xi^{z, 1}\left(R_{u}\right)\right)-\left(H_{j}\left(\Phi \circ \Xi^{z, 1}\right)\right)\left(u, R_{u}\right)\right\} \nu(d z) .
\end{aligned}
$$

Thus, applying the Itô formula implies that

$$
\begin{aligned}
\left(H_{j} \Phi\right)\left(s, R_{s}\right)= & \left(H_{j} \Phi\right)(0, r)+\int_{0}^{s} \sum_{k=1}^{m}\left(H_{k}\left(H_{j} \Phi\right)\right)\left(u, R_{u}\right) d B_{u}^{k} \\
& +\int_{0}^{s} \int_{\mathbb{R}_{0}^{m}}\left\{\left(H_{j} \Phi\right)\left(u, \Xi^{z, 1}\left(R_{u-}\right)\right)-\left(H_{j} \Phi\right)\left(u, R_{u-}\right)\right\} \tilde{N}(d u, d z) \\
& +\int_{0}^{s}\left\{\frac{\partial}{\partial u}\left(\left(H_{j} \Phi\right)\left(u, R_{u}\right)\right)+\left(\mathcal{A}\left(H_{j} \Phi\right)\right)\left(u, R_{u}\right)\right\} d u \\
= & \left(H_{j} \Phi\right)(0, r)+\int_{0}^{s} \sum_{k=1}^{m}\left(H_{k}\left(H_{j} \Phi\right)\right)\left(u, R_{u}\right) d B_{u}^{k} \\
& +\int_{0}^{s} \int_{\mathbb{R}_{0}^{m}}\left\{\left(H_{j} \Phi\right)\left(u, \Xi^{z, 1}\left(R_{u-}\right)\right)-\left(H_{j} \Phi\right)\left(u, R_{u-}\right)\right\} \tilde{N}(d u, d z) \\
& +\int_{0}^{s} \frac{1}{2} \sum_{i=1}^{m}\left(\left[H_{i} H_{i}, H_{j}\right] \Phi\right)\left(u, R_{u}\right) d u \\
& +\int_{0}^{s} \int_{\mathbb{R}_{0}^{m}}\left\{\left(H_{j} \Phi\right)\left(u, \Xi^{z, 1}\left(R_{u}\right)\right)-\left(H_{j}\left(\Phi \circ \Xi^{z, 1}\right)\right)\left(u, R_{u}\right)\right\} \nu(d z) d u
\end{aligned}
$$

for $s \in[0, t)$, from the equation (4.1). Remark that

$$
(V(f \circ \pi))(r)=\sum_{j=1}^{m}\left(\left(H_{j}^{*}(V) H_{j}\right)(f \circ \pi)\right)(r)
$$

for $V \in \mathfrak{X}(O(M))$, and that

$$
\sum_{j=1}^{m} \frac{d L_{s}^{i j}}{d s}\left(H_{j} \Phi\right)\left(s, R_{s}\right)=-\sum_{j=1}^{m} L_{s}^{i j}\left(\left[\mathcal{A}, H_{j}\right] \Phi\right)\left(s, R_{s}\right)
$$

for $1 \leq i \leq m$. Then, the Itô product formula enables us to get the assertion (4.4) for $s \in[0, t)$. Finally, taking the limit in the equality (4.4) for $s \in[0, t)$ as $s \nearrow t$ leads us to justify the equality (4.4) for $s \in[0, t]$, because of $f \in C^{2}(M ; \mathbb{R})$ with a compact support. The proof is complete. 
(Proof of Theorem 3.3.) From Lemma 4.1, the process $\left\{N_{s} ; s \in[0, t]\right\}$ defined by

$$
N_{s}=L_{s}\left(\begin{array}{c}
\left(H_{1} \Phi\right)\left(s, R_{s}\right) \\
\vdots \\
\left(H_{m} \Phi\right)\left(s, R_{s}\right)
\end{array}\right)
$$

is a martingale. Since

$$
\left(e\left(T_{t} f\right)\right)(x)=e_{x}\left(\mathbb{E}\left[f\left(X_{t}(x)\right)\right]\right)=H_{r}\left(\mathbb{E}\left[(f \circ \pi)\left(R_{t}(r)\right)\right]\right)=(H \Phi)(0, r),
$$

we have

$$
\left(e\left(T_{t} f\right)\right)(x)=N_{0}^{*}=\mathbb{E}\left[N_{t}^{*}\right] .
$$

Denote the inner product in $\mathbb{R}^{m}$ by $\langle\cdot, \cdot\rangle_{m}$. Then, the expressions (4.3) and (4.5), and the Itô product rule enable us to see that, for $v \in \mathbb{R}^{m}$,

$$
\begin{aligned}
\left\langle\left(e\left(T_{t} f\right)\right)(x)^{*}, v\right\rangle_{m} & =\left\langle\mathbb{E}\left[N_{t}\right], v\right\rangle_{m} \\
& =\left\langle\mathbb{E}\left[\frac{1}{t} \int_{0}^{t} N_{s} d s\right], v\right\rangle_{m} \\
& =\frac{1}{t} \mathbb{E}\left[\int_{0}^{t} \sum_{i, j=1}^{m} L_{s}^{i j}\left(H_{j} \Phi\right)\left(s, R_{s}(r)\right) v_{i} d s\right] \\
& =\mathbb{E}\left[\int_{0}^{t}(H \Phi)\left(s, R_{s}(r)\right) d B_{s}\left\langle\frac{1}{t} \int_{0}^{t} L_{s} d B_{s}, v\right\rangle_{m}\right] \\
& =\mathbb{E}\left[\left\{\Phi\left(t, R_{t}(r)\right)-\Phi(0, r)\right\}\left\langle\frac{1}{t} \int_{0}^{t} L_{s} d B_{s}, v\right\rangle_{m}\right] \\
& =\mathbb{E}\left[f\left(X_{t}(x)\right)\left\langle\frac{1}{t} \int_{0}^{t} L_{s} d B_{s}, v\right\rangle_{m}\right] \\
& =\left\langle\mathbb{E}\left[f\left(X_{t}(x)\right) \frac{I_{t}^{*}}{t}\right], v\right\rangle_{m} .
\end{aligned}
$$

Here, the fourth equality can be justified by the computation of the quadratic variation on stochastic integrals with respect to the Brownian motions, while the fifth equality is just the result by the Itô product rule and (4.3). The proof of Theorem 3.3 is complete.

\subsection{Proof of Theorem 3.10}

Here, we shall give the proof of Theorem 3.10. Assume Assumptions 3.1 and 3.5 on the measure $\nu(d z)$, and Assumption 3.7 on the manifold $M$, throughout this subsection. Let us introduce the following notations:

$$
\begin{aligned}
& \hat{A}_{t}=\int_{0}^{t} \int_{\mathbb{R}_{0}^{m}} h(s, z) \hat{N}(d s, d z), \quad \tilde{A}_{t}=\int_{0}^{t} \int_{\mathbb{R}_{0}^{m}} h(s, z) \tilde{N}(d s, d z), \\
& L_{t}=\int_{0}^{t} \int_{\mathbb{R}_{0}^{m}}\left\{\Phi\left(s, \Xi^{z, 1}\left(R_{s-}\right)\right)-\Phi\left(s, R_{s-}\right)\right\} N(d s, d z), \\
& \hat{L}_{t}=\int_{0}^{t} \int_{\mathbb{R}_{0}^{m}}\left\{\Phi\left(s, \Xi^{z, 1}\left(R_{s-}\right)\right)-\Phi\left(s, R_{s-}\right)\right\} \hat{N}(d s, d z), \\
& \tilde{L}_{t}=\int_{0}^{t} \int_{\mathbb{R}_{0}^{m}}\left\{\Phi\left(s, \Xi^{z, 1}\left(R_{s-}\right)\right)-\Phi\left(s, R_{s-}\right)\right\} \tilde{N}(d s, d z) .
\end{aligned}
$$

Our strategy to attack the main interest in this section is almost parallel to the method on the Euclidean space studied in [15]. 


\section{Lemma 4.2.}

$$
\mathbb{E}\left[f\left(X_{t}\right) A_{t}\right]=\mathbb{E}\left[\int_{0}^{t} \int_{\mathbb{R}_{0}^{m}} \Phi\left(s, \Xi^{z, 1}\left(R_{s}\right)\right) h(s, z) \hat{N}(d s, d z)\right] .
$$

Proof. Write $W_{t}:=\int_{0}^{t}(H \Phi)\left(s, R_{s}\right) d B_{s}$. Then, the Itô product formula yields that

$$
\begin{aligned}
\mathbb{E}\left[W_{t} A_{t}\right]= & \mathbb{E}\left[\int_{0}^{t} \int_{\mathbb{R}_{0}^{m}} W_{s} h(s, z) \hat{N}(d s, d z)\right], \\
\mathbb{E}\left[\tilde{L}_{t} A_{t}\right]= & \mathbb{E}\left[\int_{0}^{t} \int_{\mathbb{R}_{0}^{m}} \tilde{L}_{s} h(s, z) \hat{N}(d s, d z)\right] \\
& +\mathbb{E}\left[\int_{0}^{t} \int_{\mathbb{R}_{0}^{m}}\left(\Phi\left(s, \Xi^{z, 1}\left(R_{s}\right)\right)-\Phi\left(s, R_{s}\right)\right) h(s, z) \hat{N}(d s, d z)\right] .
\end{aligned}
$$

Since $f\left(X_{t}\right)=\mathbb{E}\left[f\left(X_{t}\right)\right]+W_{t}+\tilde{L}_{t}$ from (4.3), we have

$$
\begin{aligned}
\mathbb{E}\left[f\left(X_{t}\right) A_{t}\right]= & \mathbb{E}\left[\mathbb{E}\left[f\left(X_{t}\right)\right] A_{t}\right]+\mathbb{E}\left[W_{t} A_{t}\right]+\mathbb{E}\left[\tilde{L}_{t} A_{t}\right] \\
= & \mathbb{E}\left[f\left(X_{t}\right)\right] \mathbb{E}\left[A_{t}\right]+\mathbb{E}\left[\int_{0}^{t} \int_{\mathbb{R}_{0}^{m}} W_{s} h(s, z) \hat{N}(d s, d z)\right] \\
& +\mathbb{E}\left[\int_{0}^{t} \int_{\mathbb{R}_{0}^{m}} \tilde{L}_{s} h(s, z) \hat{N}(d s, d z)\right] \\
& +\mathbb{E}\left[\int_{0}^{t} \int_{\mathbb{R}_{0}^{m}}\left\{\Phi\left(s, \Xi^{z, 1}\left(R_{s}\right)\right)-\Phi\left(s, R_{s}\right)\right\} h(s, z) \hat{N}(d s, d z)\right] \\
= & \mathbb{E}\left[f\left(X_{t}\right) \hat{A}_{t}\right]-\mathbb{E}\left[\int_{0}^{t} \int_{\mathbb{R}_{0}^{m}}\left(W_{t}-W_{s}\right) h(s, z) \hat{N}(d s, d z)\right] \\
& -\mathbb{E}\left[\int_{0}^{t} \int_{\mathbb{R}_{0}^{m}}\left(\tilde{L}_{t}-\tilde{L}_{s}\right) h(s, z) \hat{N}(d s, d z)\right] \\
& +\mathbb{E}\left[\int_{0}^{t} \int_{\mathbb{R}_{0}^{m}}\left(\Phi\left(s, \Xi^{z, 1}\left(R_{s}\right)\right)-\Phi\left(s, R_{s}\right)\right) h(s, z) \hat{N}(d s, d z)\right] \\
= & I_{1, t}+I_{2, t}+I_{3, t}+I_{4, t} .
\end{aligned}
$$

As for $I_{1, T}$, since the process $\left\{\Phi\left(s, R_{s}\right) ; s \in[0, t]\right\}$ is a martingale from (4.2) and (4.3), we have

$$
\begin{aligned}
I_{1, t} & =\mathbb{E}\left[\Phi\left(t, R_{t}\right) \int_{0}^{t} \int_{\mathbb{R}_{0}^{m}} h(s, z) \hat{N}(d s, d z)\right] \\
& =\int_{0}^{t} \int_{\mathbb{R}_{0}^{m}} \mathbb{E}\left[\mathbb{E}\left[\Phi\left(t, R_{t}\right) \mid \mathcal{F}_{s}\right] h(s, z)\right] \hat{N}(d s, d z) \\
& =\int_{0}^{t} \int_{\mathbb{R}_{0}^{m}} \mathbb{E}\left[\Phi\left(s, R_{s}\right) h(s, z)\right] \hat{N}(d s, d z) \\
& =\mathbb{E}\left[\int_{0}^{t} \int_{\mathbb{R}_{0}^{m}} \Phi\left(s, R_{s}\right) h(s, z) \hat{N}(d s, d z)\right] .
\end{aligned}
$$


As for $I_{2, t}$ and $I_{3, t}$, since $W=\left\{W_{s} ; s \in[0, t]\right\}$ and $\tilde{L}=\left\{\tilde{L}_{s} ; s \in[0, t]\right\}$ are martingales, we see that

$$
\begin{aligned}
& I_{2, t}=-\int_{0}^{t} \int_{\mathbb{R}_{0}^{m}} \mathbb{E}\left[\mathbb{E}\left[W_{t}-W_{s} \mid \mathcal{F}_{s}\right] h(s, z)\right] \hat{N}(d s, d z)=0, \\
& I_{3, t}=-\int_{0}^{t} \int_{\mathbb{R}_{0}^{m}} \mathbb{E}\left[\mathbb{E}\left[\tilde{L}_{t}-\tilde{L}_{s} \mid \mathcal{F}_{s}\right] h(s, z)\right] \hat{N}(d s, d z)=0 .
\end{aligned}
$$

Therefore, we can get that

$$
\mathbb{E}\left[f\left(X_{t}\right) A_{t}\right]=I_{1, t}+I_{4, t}=\mathbb{E}\left[\int_{0}^{t} \int_{\mathbb{R}_{0}^{m}} \Phi\left(s, \Xi^{z, 1}\left(R_{s}\right)\right) h(s, z) \hat{N}(d s, d z)\right] .
$$

The proof is complete.

Lemma 4.3.

$$
\mathbb{E}\left[e_{x}\left(f\left(X_{t}(x)\right)\right) A_{t}\right]=-\mathbb{E}\left[f\left(X_{t}(x)\right) J_{t}\right] .
$$

Proof. Remark that

$$
\begin{aligned}
& \mathbb{E}\left[\int_{0}^{t} \int_{\mathbb{R}_{0}^{m}} \Phi\left(s, \Xi^{z, 1}\left(R_{s}\right)\right) e_{x}(h(s, z)) \hat{N}(d s, d z)\right] \\
& =\mathbb{E}\left[f\left(X_{t}\right) \int_{0}^{t} \int_{\mathbb{R}_{0}^{m}} e_{x}(h(s, z)) N(d s, d z)\right] \\
& =\mathbb{E}\left[f\left(X_{t}\right) e_{x}\left(A_{t}\right)\right]
\end{aligned}
$$

similarly to Lemma 4.2. Furthermore, by using the equality (4.3) and the divergence formula on the usual integral over $\mathbb{R}_{0}^{m}$, we see that

$$
\begin{aligned}
\mathbb{E}\left[f\left(X_{t}\right) J_{t}\right] & =\mathbb{E}\left[\tilde{L}_{t} \int_{0}^{t} \int_{\mathbb{R}_{0}^{m}} \frac{\operatorname{div}_{z}(g(z) v(s, z))}{g(z)} \tilde{N}(d s, d z)\right] \\
& =\mathbb{E}\left[\int_{0}^{t} \int_{\mathbb{R}_{0}^{m}}\left\{\Phi\left(s, \Xi^{z, 1}\left(R_{s}\right)\right)-\Phi\left(s, R_{s}\right)\right\} \operatorname{div}_{z}(g(z) v(s, z)) d z d s\right] \\
& =-\mathbb{E}\left[\int_{0}^{t} \int_{\mathbb{R}_{0}^{m}} \partial_{z}\left(\Phi\left(s, \Xi^{z, 1}\left(R_{s}\right)\right) v(s, z) \hat{N}(d s, d z)\right]\right. \\
& =-\mathbb{E}\left[\int_{0}^{t} \int_{\mathbb{R}_{0}^{m}} H_{r}\left(\Phi\left(s, \Xi^{z, 1}\left(R_{s}(r)\right)\right) h(s, z) \hat{N}(d s, d z)\right] .\right.
\end{aligned}
$$

Hence, Lemma 4.2 enables us to get

$$
\begin{aligned}
\mathbb{E}\left[e_{x}\left(f\left(X_{t}\right)\right) A_{t}\right]= & \mathbb{E}\left[e_{x}\left(f\left(X_{t}\right) A_{t}\right)\right]-\mathbb{E}\left[f\left(X_{t}\right) e_{x}\left(A_{t}\right)\right] \\
= & e_{x}\left(\mathbb{E}\left[f\left(X_{t}\right) A_{t}\right]\right)-\mathbb{E}\left[\int_{0}^{t} \int_{\mathbb{R}_{0}^{m}} \Phi\left(s, \Xi^{z, 1}\left(R_{s}\right)\right) e_{x}(h(s, z)) \hat{N}(d s, d z)\right] \\
= & e_{x}\left(\mathbb{E}\left[\int_{0}^{t} \int_{\mathbb{R}_{0}^{m}} \Phi\left(s, \Xi^{z, 1}\left(R_{s}\right)\right) h(s, z) \hat{N}(d s, d z)\right]\right) \\
& -\mathbb{E}\left[\int_{0}^{t} \int_{\mathbb{R}_{0}^{m}} \Phi\left(s, \Xi^{z, 1}\left(R_{s}\right)\right) e_{x}(h(s, z)) \hat{N}(d s, d z)\right]
\end{aligned}
$$




$$
\begin{aligned}
& =\mathbb{E}\left[\int_{0}^{t} \int_{\mathbb{R}_{0}^{m}} e_{x}\left(\Phi\left(s, \Xi^{z, 1}\left(R_{s}\right)\right)\right) h(s, z) \hat{N}(d s, d z)\right] \\
& =\mathbb{E}\left[\int_{0}^{t} \int_{\mathbb{R}_{0}^{m}} H_{r}\left(\Phi\left(s, \Xi^{z, 1}\left(R_{s}(r)\right)\right)\right) h(s, z) \hat{N}(d s, d z)\right] \\
& =-\mathbb{E}\left[f\left(X_{t}\right) J_{t}\right],
\end{aligned}
$$

which completes the proof.

(Proof of Theorem 3.10.) Recall the definition (3.4) of $M_{t}^{\lambda}$ in Remark 3.11. For each $\lambda>0$, we shall define

$$
\left.\frac{d \mathbb{P}^{\lambda}}{d \mathbb{P}}\right|_{\mathcal{F}_{t}}:=\exp \left(-\lambda A_{t}-M_{t}^{\lambda}\right)
$$

Since the $[0,+\infty)$-valued function $h$ is deterministic, the Girsanov theorem for jump processes tells us to see that $N(d s, d z)$ is also the Poisson random measure with the intensity

$$
\hat{N}^{\lambda}(d s, d z):=\exp (-\lambda h(t, z)) \hat{N}(d t, d z)
$$

under the new probability measure $d \mathbb{P}^{\lambda}$, which can be checked easily as a routine work by the application of the Itô formula.

Since

$$
\frac{\operatorname{div}_{z}(\exp (-\lambda h(s, z)) g(z) v(s, z))}{\exp (-\lambda h(s, z)) g(z)}=\frac{\operatorname{div}_{z}(g(z) v(s, z))}{g(z)}-\lambda \partial_{z} h(s, z) v(s, z),
$$

we have

$$
\begin{aligned}
J_{t}^{\lambda} & :=\int_{0}^{t} \int_{\mathbb{R}_{0}^{m}} \frac{\operatorname{div}_{z}(\exp (-\lambda h(s, z)) g(z) v(t, z))}{\exp (-\lambda h(s, z)) g(z)} \tilde{N}^{\lambda}(d s, d z) \\
& =J_{t}-\lambda K_{t},
\end{aligned}
$$

where $\tilde{N}^{\lambda}(d s, d z)=N(d s, d z)-\hat{N}^{\lambda}(d s, d z)$. Applying Lemma 4.3 to the measure $d \mathbb{P}^{\lambda}$ implies that

$$
\mathbb{E}^{\lambda}\left[e_{x}\left(f\left(X_{T}\right)\right) A_{T}\right]=-\mathbb{E}^{\lambda}\left[f\left(X_{t}\right) J_{t}^{\lambda}\right] .
$$

Since the $[0,+\infty)$-valued function $h$ is deterministic, we see that

$$
\begin{aligned}
\mathbb{E}\left[e_{x}\left(f\left(X_{T}\right)\right)\right] & =\int_{0}^{\infty} \mathbb{E}^{\lambda}\left[e_{x}\left(f\left(X_{T}\right)\right) A_{T}\right] \exp \left(M_{T}^{\lambda}\right) d \lambda \\
& =\int_{0}^{\infty} \mathbb{E}^{\lambda}\left[f\left(X_{t}\right)\left(-J_{t}+\lambda K_{t}\right)\right] \exp \left(M_{T}^{\lambda}\right) d \lambda \\
& =\mathbb{E}\left[f\left(X_{t}\right)\left(-\frac{J_{t}}{A_{t}}+\frac{K_{t}}{A_{t}^{2}}\right)\right],
\end{aligned}
$$

which completes the proof of Theorem 3.10.

\subsection{Proof of Theorem 3.12}

In this subsection, let us give the proof of Theorem 3.12. Since our strategy to attack the problem is almost parallel to Subsection 4.2 , we shall explain its sketch, only.

(Proof of Theorem 3.12.) Recall the function $M_{t}^{\lambda}$ given by (3.4) in Remark 3.11, and the probability measure $\mathbb{P}^{\lambda}$ defined by (4.8) in Subsection 4.2 . Then, similarly to the arguments discussed in Subsections 4.1 and 4.2, we can obtain that

$$
\mathbb{E}^{\lambda}\left[e_{x}\left(f\left(X_{t}(x)\right)\right)\left(t+A_{t}\right)\right]=\mathbb{E}^{\lambda}\left[f\left(X_{t}(x)\right)\left\{I_{t}-J_{t}+\lambda K_{t}\right\}\right]
$$


Hence, similarly to the proof of Theorem 3.10, we see that

$$
\begin{aligned}
e_{x}\left(\mathbb{E}\left[f\left(X_{t}\right)\right]\right) & =\mathbb{E}\left[e_{x}\left(f\left(X_{t}\right)\right)\left(t+A_{t}\right) \int_{0}^{\infty} \exp \left\{-\lambda\left(t+A_{t}\right)\right\} d \lambda\right] \\
& =\int_{0}^{\infty} \mathbb{E}^{\lambda}\left[e_{x}\left(f\left(X_{t}\right)\right)\left(t+A_{t}\right)\right] \exp \left(-\lambda t+N_{t}^{\lambda}\right) d \lambda \\
& =\int_{0}^{\infty} \mathbb{E}^{\lambda}\left[f\left(X_{t}\right)\left(I_{t}-J_{t}+\lambda K_{t}\right)\right] \exp \left(-\lambda t+N_{t}^{\lambda}\right) d \lambda \\
& =\mathbb{E}\left[f\left(X_{t}\right)\left(\frac{I_{t}-J_{t}}{t+A_{t}}+\frac{K_{t}}{\left(t+A_{t}\right)^{2}}\right)\right] .
\end{aligned}
$$

The proof of Theorem 3.12 is complete.

\section{References}

[1] Applebaum, D. and Estrade, A.: Isotropic Lévy processes on Riemannian manifolds, Ann. Probab. 28 (2000), 166-184. MR-1756002

[2] Applebaum, D. and Kunita, H.: Lévy flows on manifolds and Lévy processes on Lie groups, J. Math. Kyoto Univ. 33 (1993), 1103-1123. MR-1251218

[3] Bismut, J. M.: Martingales, the Malliavin calculus and hypoellipticity under general Hörmander's condition, Z. für Wahrscheinlichkeitstheorie verw. Gebiete, 63 (1981), 469-505. MR-0621660

[4] Elworthy, K. D. and Li, X.-M.: Formulae for the derivatives of heat semigroups, J. Funct. Anal., 125 (1994), 252-286. MR-1297021

[5] Fujiwara, T.: Stochastic differential equations of jump type on manifolds and Lévy flows, $J$. Math. Kyoto Univ., 31 (1991), 99-119. MR-1093330

[6] Hsu, E.: Stochastic Analysis on Manifolds, American Mathematical Society, 2002. 281 pp. MR-1882015

[7] Hunt, G. A.: Semigroups of measures on Lie groups, Trans. Amer. Math. Soc., 81 (1956), 264-293. MR-0079232

[8] Ikeda, N. and Watanabe, S.: Stochastic Differential Equations and Diffusion Processes, 2nd edition, North-Holland/Kodansha, 1989. 464 pp. MR-1011252

[9] Ishikawa, Y.: Stochastic Calculus of Variations: For Jump Processes 2nd edition, De Gruyter, 2016. 278 pp. MR-3495001

[10] Kobayashi, S. and Nomizu, K.: Foundations of Differential Geometry. Volume I, Wiley, New York, 1963. 348 pp. MR-1393940

[11] Kunita, H.: Stochastic Flows and Jump-Diffusions, Springer, 2019. 352 pp. MR-3929750

[12] Nualart, D.: The Malliavin Calculus and Related Topics, 2nd edition, Springer, Berlin, 2006. 382 pp. MR-2200233

[13] Sakai, T.: Riemannian Geometry, American Mathematical Society, 1996. 358 pp. MR-1390760

[14] Takeuchi, A.: The Malliavin calculus for SDE with jumps and the partially hypoelliptic problem, Osaka J. Math., 39 (2002), 523-559. MR-1932281

[15] Takeuchi, A.: The Bismut-Elworthy-Li type formulae for stochastic differential equations with jumps, J. Theor. Probab. 23 (2010), 576-604. MR-2644877

[16] Taniguchi, S.: Malliavin's stochastic calculus of variations for manifold-valued Wiener functionals and its applications, Z. Wahrsch. Verw. Gebiete, 65 (1983), 269-290. MR-0722132

Acknowledgments. The authors would like to thank Professor Masamichi Yoshida for giving us some valuable comments. 


\section{Electronic Journal of Probability Electronic Communications in Probability}

\section{Advantages of publishing in EJP-ECP}

- Very high standards

- Free for authors, free for readers

- Quick publication (no backlog)

- Secure publication $\left(\mathrm{LOCKSS}^{1}\right)$

- Easy interface (EJMS²)

\section{Economical model of EJP-ECP}

- Non profit, sponsored by $\mathrm{IMS}^{3}, \mathrm{BS}^{4}$, ProjectEuclid ${ }^{5}$

- Purely electronic

\section{Help keep the journal free and vigorous}

- Donate to the IMS open access fund ${ }^{6}$ (click here to donate!)

- Submit your best articles to EJP-ECP

- Choose EJP-ECP over for-profit journals

\footnotetext{
${ }^{1}$ LOCKSS: Lots of Copies Keep Stuff Safe http://www. lockss.org/

${ }^{2}$ EJMS: Electronic Journal Management System http://www.vtex.lt/en/ejms.html

${ }^{3}$ IMS: Institute of Mathematical Statistics http://www.imstat.org/

${ }^{4}$ BS: Bernoulli Society http://www. bernoulli-society.org/

${ }^{5}$ Project Euclid: https://projecteuclid.org/

${ }^{6}$ IMS Open Access Fund: http://www.imstat.org/publications/open.htm
} 